\title{
Investigation of EGFR mutations in non-small cell lung cancer usually undetectable by PCR methods
}

\author{
TAISUKE MATSUBARA ${ }^{1}$, EIJI NAKAJIMA ${ }^{2}$, HARUKA NAMIKAWA ${ }^{1}$, SHOTARO ONO $^{2}$, IKKI TAKADA ${ }^{2}$, \\ TATSUO OHIRA $^{1}$, YUKIO MORISHITA ${ }^{3}$, TERUO MIYAZAKI ${ }^{4}$, KINYA FURUKAWA $^{2}$ and NORIHIKO IKEDA ${ }^{1}$ \\ ${ }^{1}$ Department of Surgery, Tokyo Medical University, Tokyo 160-0023; \\ Departments of ${ }^{2}$ Thoracic Surgery, ${ }^{3}$ Diagnostic Pathology and ${ }^{4}$ Joint Research Center, \\ Tokyo Medical University Ibaraki Medical Center, Ami, Ibaraki 300-0395, Japan
}

Received July 15, 2021; Accepted September 10, 2021

DOI: $10.3892 / \mathrm{mco} .2021 .2447$

\begin{abstract}
Epidermal growth factor receptor (EGFR) mutations are the most significant genomic drivers of non-small cell lung cancer (NSCLC) and determine the efficacy of EGFR tyrosine kinase inhibitor (EGFR-TKI) therapy. PCR methods are used clinically for the detection of EGFR mutations. The Scorpion Amplification Refractory Mutation System (Scorpion-ARMS) and the cobas ${ }^{\circledR}$ EGFR Mutation Test v2 (cobas v2) are widely used PCR methods. However, those PCR methods only selectively detect the common EGFR mutations. The aim of the present study was to reveal the true frequency of EGFR mutations in NSCLC by investigating EGFR mutations usually undetectable by PCR methods by using direct sequencing. A total of 70 Japanese patients who underwent lung resection for NSCLC between September 2016 and March 2019 were included in the present study. Subsequently, PCR methods and direct sequencing were performed. In total, 29 mutations were detected by cobas v2. In total, 41 patients were identified as EGFR wild-type by cobas v2, among whom direct sequencing detected mutations in 3 patients. Subsequent Scorpion-ARMS was performed in the 3 patients in whom direct sequencing detected mutations. In total, one exon 21 L858R + G863D compound mutation was identified as a L858R single mutation, and two other mutations were undetectable. Moreover, 1 patient who was 'wild-type' on cobas v2 but 'EGFR mutation' on direct
\end{abstract}

Correspondence to: Dr Taisuke Matsubara, Department of Surgery, Tokyo Medical University, 6-7-1 Nishishinjuku, Shinjuku, Tokyo 160-0023, Japan

E-mail: t-matsu@tokyo-med.ac.jp

Abbreviations: TKI, tyrosine kinase inhibitor; NSCLC, non-small cell lung cancer; FFPE, formalin-fixed and paraffin embedded; Scorpion-ARMS, Scorpion Amplification Refractory Mutation System; LOD, limit of detection; PET/CT, positron emission tomography/CT

Key words: non-small cell lung cancer, EGFR mutation, PCR method, LOD, TKI sequencing developed recurrence after surgery and responded to EGFR-TKI treatment. In present study, the percentage of undetectable EGFR mutations by cobas v2 was $9.4 \%$ in 32 mutations. It was inferred that the cause of the discrepancy in the mutation type (L858R + G863D in exon 21, and L858R in exon 21) between cobas v2 and Scorpion ARMS was due to the different limit of detection between these two PCR methods. In conclusion, the findings of the present study suggested that a selective mutation detection method may decrease the opportunity of patients with NSCLC to receive EGFR-TKI therapy. Thus, the development of a screening test to determine the EGFR status as wild-type or mutant is required for EGFR-TKI therapy.

\section{Introduction}

The presence of epidermal growth factor receptor (EGFR) mutations determines the efficacy of EGFR tyrosine kinase inhibitor (EGFR-TKI) therapy in patients with non-small cell lung cancer (NSCLC). EGFR-TKIs are used as the first-line therapy for advanced NSCLC cases with EGFR mutations (1-4). Therefore, detecting these mutations is crucial for patients with NSCLC. Previous clinical studies have demonstrated favorable outcomes of patients with common mutations receiving EGFR-TKI therapy, such as those with selected exon 19 deletions and a L858R point mutation in exon 21 (1-4). In EGFR exons 18-21, the frequency of common mutations is 90\% among all EGFR mutations. The remaining $10 \%$ includes uncommon mutations, which are a heterogeneous group of molecular alterations (4-6). The variant mutations consist of $>400$ types, which were registered in Catalogue of Somatic Mutation in Cancer database version 84 in 2018 (6). The response to EGFR-TKIs in cases with uncommon mutations has been debated, but remains largely unknown at present.

PCR methods are used clinically for the detection of EGFR mutations. Two widely used PCR methods have been approved as in vitro diagnostic methods, namely the Scorpion Amplification Refractory Mutation System (Scorpion-ARMS; Qiagen therascreen ${ }^{\circledR}$ EGFR; Qiagen, Inc.) and the cobas ${ }^{\circledR}$ EGFR Mutation Test v2 (cobas v2; Roche Diagnostics). These methods are beneficial for clinical use due to their short detection time and cost-effectiveness. 
However, these PCR methods only selectively detect certain EGFR mutations, such as exon 19 deletions and a L858R point mutation in exon 21 , and not others $(7,8)$. Therefore, the aim of the present study was to identify the frequency of the mutations not detected by these PCR methods. To achieve this aim, the current study evaluated EGFR mutations using PCR methods and direct sequencing.

\section{Patients and methods}

Patients. The present study included 70 Japanese patients with NSCLC, from whom informed consent was obtained for the use of their samples in this research. These patients underwent surgical lung resection at the Tokyo Medical University Ibaraki Medical Center (Ami, Japan) between September 2016 and March 2019 (Table I). A total of $61.4 \%$ of the patients were male and $38.6 \%$ were female. The patient age ranged between 42 and 84 years. PCR methods and direct sequencing were performed to analyze EGFR status in the surgical samples. EGFR-TKIs were administered to the patients who developed NSCLC recurrence after surgery. The protocol of the present study received ethics approval from the Institutional Review Board of Tokyo Medical University (Tokyo, Japan; approval no. 17-56).

PCR method as the first EGFR mutation testing method. All 70 surgical samples were formalin-fixed and paraffin-embedded (FFPE). This PCR method was performed with cobas v2, which is an allele-specific real-time PCR system used to identify 42 types of EGFR nucleotide mutations in exons 18-21 using FFPE NSCLC tissues. According to the package insert, cobas v2 requires $\geq 3.13$ ng DNA, which includes $5 \%$ mutated DNA, to detect the mutation. DNA isolation, amplification/detection and result reporting can be performed in $<8 \mathrm{~h}$, with up to 30 specimens processed simultaneously (7). DNA was subjected to cobas v2 at SRL, Inc.

DNA extraction and direct sequencing analysis as the second EGFR testing method. Direct sequencing was performed to further analyze the presence of EGFR mutations in the FFPE surgical samples determined as wild-type EGFR by cobas v2. Tumor cells were harvested via macrodissection, and DNA was extracted from tumor cells using a DNeasy Tissue kit (Qiagen, Inc.). PCR was performed using a primer set flanking exons 18-21 as the TKI domain. PCR products of different sizes were treated with ExoSAP-IT ${ }^{\mathrm{TM}}$ PCR Product Cleanup (Thermo Fisher Scientific, Inc.) according to the manufacture's protocol. The PCR primers were as fllows: Exon 18: Forward, 5'-TGAGGTGACCCTTGTCTCTG-3' and reverse, 5'-CCTGTGCCAGGGACCTTAC-3'(180 nucleotides); exon 19: Forward, 5'-ATGTGGCACCATCTCACAAT-3' and reverse, 5'-CCACACAGCAAAGCAGAAAC-3' (178 nucleotides); exon 20: Forward, 5'-ATGCGAAGCCACACTGAC-3' and reverse, 5'-CCTTCCCTGATTACCTTTGC-3' (238 nucleotides); and exon 21: Forward, 5'-GCAGGGTCTTCTCYGTTTCA-3' and reverse, 5'-TGACCTAAAGCCACCTCCTT-3' (199 nucleotides). These kits were used according to the manufacturer's instructions. Sequencing analysis was performed using an Applied Biosystems ${ }^{\mathrm{TM}}$ 3130xl Genetic Analyzer (Thermo Fisher Scientific, Inc.) according to the manufacturer's protocol.
PCR method as the third EGFR mutation testing method. Scorpion-ARMS, which is a real time-PCR assay that combines the ARMS and Scorpions fluorescent primer/probe system, was performed to further validate the samples shown as 'wild-type' on cobas v2 but 'EGFR mutations' on direct sequencing. Scorpion-ARMS can detect 29 somatic mutations in exons 18-21 of EGFR. The limit of detection (LOD) is $\sim 1 \%$ (8). DNA was subjected to Scorpion-ARMS at SRL, Inc.

EGFR-TKI treatment for NSCLC recurrence after surgery. EGFR-TKIs were administered to patients who developed recurrence after surgery. The determination of the efficacy of EGFR-TKI therapy was based on the Response Evaluation Criteria in Solid Tumors (RECIST) (9) using CT, and Positron Emission Tomography (PET) Response Criteria In Solid Tumors (PERCIST) (10) using fluorodeoxyglucose (FDG) uptake on PET/CT. The cases with partial or complete response were confirmed through changes of tumor measurements, according to RECIST. The cases with complete metabolic response were confirmed using the $100 \%$ standardized uptake value (SUV) normalized to lean body mass (LBM) decline, according to PERCIST. In PERCIST, the LBM-corrected SUV (SUL) is proposed as a more appropriate quantitative method. Fat contributes to body weight (BW), and the accumulation of FDG and the SUV are affected by BW. The SUL was calculated according to the Janmahasatian formula (11). Stable disease was defined as fulfilling the criteria for $\geq 8$ weeks following the start of EGFR-TKI therapy. Patients receiving treatment for $>4$ weeks were considered as assessable, and patients who had received EGFR-TKI therapy for $<4$ weeks were considered as non-assessable.

\section{Results}

EGFR mutation status. The first PCR method, cobas v2, detected 29 mutations among all 70 patients with NSCLC. The remaining 41 patients were identified as NSCLC with EGFR wild-type by cobas v2, among whom direct sequencing as the second EGFR mutation testing method detected mutations in 3 patients (Table II). These 3 cases were validated using Scorpion-ARMS as the third EGFR mutation testing method. L858R + G863D compound mutations in direct sequencing (Fig. 1) were identified as a L858R single mutation, and the others were identified as wild-type by Scorpion-ARMS. Thus, two mutations were undetectable by both PCR methods (Table II).

EGFR-TKI treatment for NSCLC recurrence after surgery. Among the 32 patients with EGFR mutations, EGFR-TKIs were administered to 3 patients with postoperative recurrence. In total, one patient with an exon 19 deletion detected by cobas v2 partially responded to osimertinib (third-generation EGFR-TKI), according to RECIST. Another patient with exon $21 \mathrm{~L} 858 \mathrm{R}+\mathrm{L} 861 \mathrm{Q}$ detected by cobas v2 was treated with gefitinib (first-generation EGFR-TKI) but was not deemed as assessable due to the development of an adverse event (interstitial lung disease). The other patient with exon 21 L858R + G863D detected by direct sequencing responded to afatinib (second-generation EGFR-TKI); this was the case shown as 'wild-type' on cobas v2 but 'EGFR mutation' on 
Table I. Background information of the 70 patients with non-small cell lung cancer.

Variables

Age, years [median (range)]

Sex, male/female

Histology, ADC/SCC/LCC/PC

Pathological stage, 0/I/II/III/IV

Smoking status, never/former + current
Total, n $(\%)$

$43(61.4) / 27(38.6)$

$61(87.1) / 6(8.6) / 1(1.4) / 2(2.9)$

$5(7.1) / 41(58.6) / 14(20.0) / 5(7.2) / 5(7.1)$

$26(37.1) / 44(62.9)$

ADC, adenocarcinoma; SCC, squamous cell carcinoma; LCC, large cell carcinoma; PC, pleomorphic carcinoma.

Table II. Information on 32 EGFR mutations.

\begin{tabular}{|c|c|c|c|c|}
\hline Exon/amino acid & No. & cobas v2 & Direct sequencing & Scorpion ARMS \\
\hline \multicolumn{5}{|l|}{ Exon 18} \\
\hline G719X & 1 & 1 & & \\
\hline Exon 19 deletion & 12 & 12 & & \\
\hline \multicolumn{5}{|l|}{ Exon 20} \\
\hline H773_V774 ins TH & 1 & Wild-type & 1 & Wild-type \\
\hline \multicolumn{5}{|l|}{ Exon 21} \\
\hline L833V + H835L & 1 & Wild-type & 1 & Wild-type \\
\hline L858R & 14 & 14 & & \\
\hline L858R + L861Q & 1 & 1 & & \\
\hline L858R + G863D & 1 & Wild-type & 1 & $\mathrm{~L}^{858 \mathrm{R}^{\mathrm{a}}}$ \\
\hline L861Q & 1 & 1 & & \\
\hline Total EGFR mutations & 32 & 29 & 3 & 1 \\
\hline
\end{tabular}

${ }^{\mathrm{a}} \mathrm{L} 858 \mathrm{R}+$ G863D was identified as L858R by Scorpion-ARMS. EGFR, epidermal growth factor receptor; ins, insertion; Scorpion-ARMS, Scorpion Amplification Refractory Mutation System; cobas v2, cobas ${ }^{\circledR}$ EGFR Mutation Test v2.

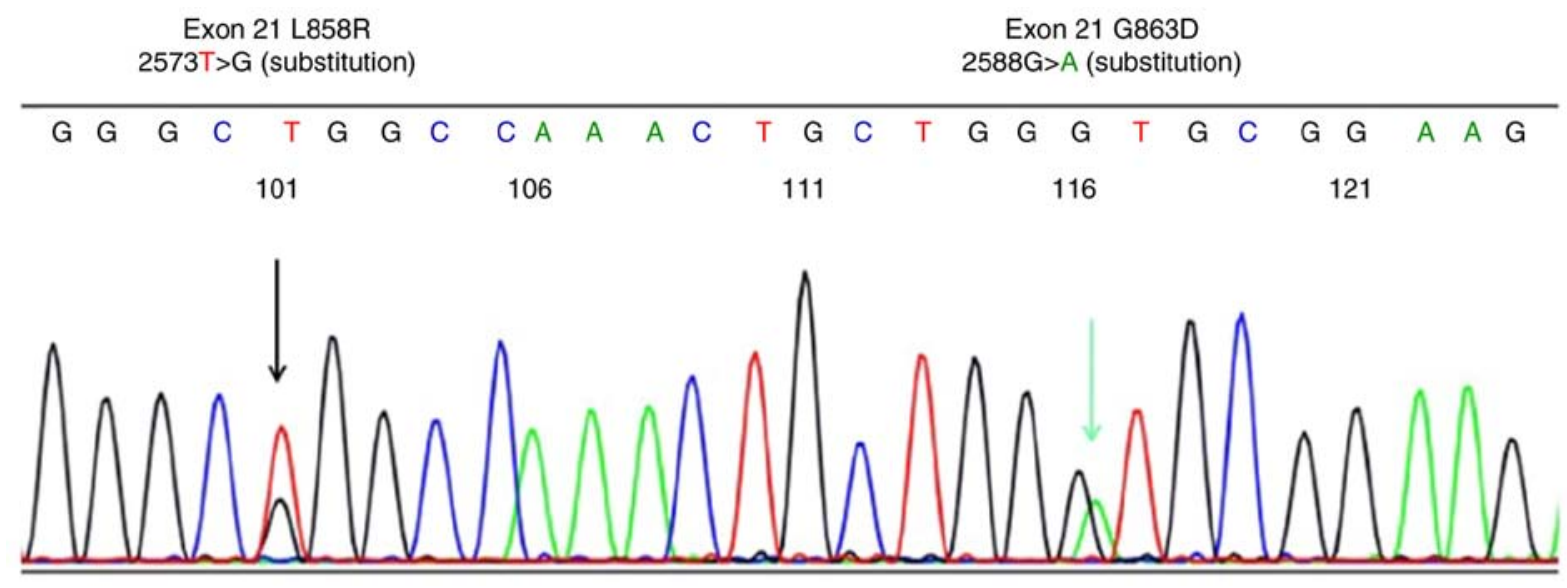

Figure 1. Sequence chromatogram showing the L858R + G863D compound mutation in EGFR exon 21. The amino acid changes corresponded to the codon alternations as follows: L858R (CTG $\rightarrow$ CGG) in exon 21; G863D (GGT $\rightarrow$ GAT) in exon 21.

direct sequencing. At recurrence, SUL was $>5.0$ in the right supraclavicular lymph node and the right pleural dissemination, as detected via PET/CT (Fig. 2A and B). Moreover, the level of carcinoembryonic antigen (CEA), a tumor marker of lung adenocarcinoma, was increased to $28.3 \mathrm{ng} / \mathrm{ml}$ (normal reference value $<5.0 \mathrm{ng} / \mathrm{ml}$ ). After EGFR-TKI therapy, a complete metabolic response was observed, as evident by the FDG uptake on PET/CT, according to PERCIST (Fig. 2C and D), and CEA levels decreased to $4.3 \mathrm{ng} / \mathrm{ml}$, which was within the normal range. 
A

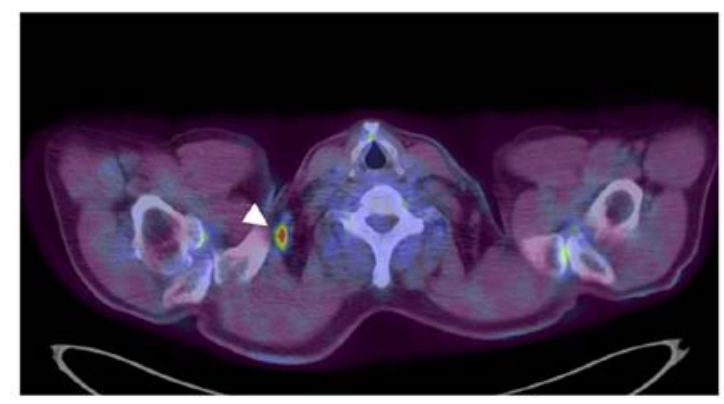

C

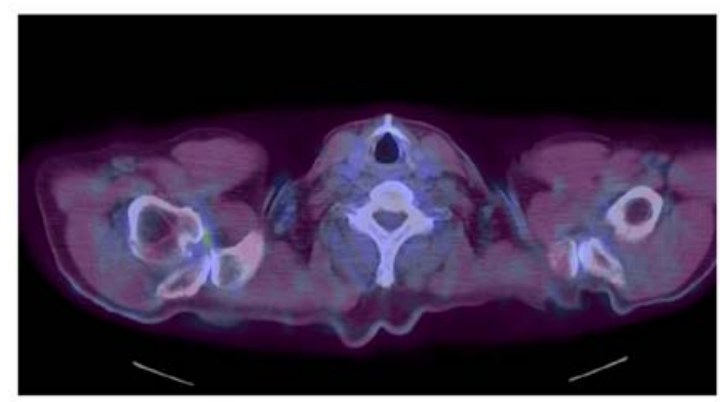

B

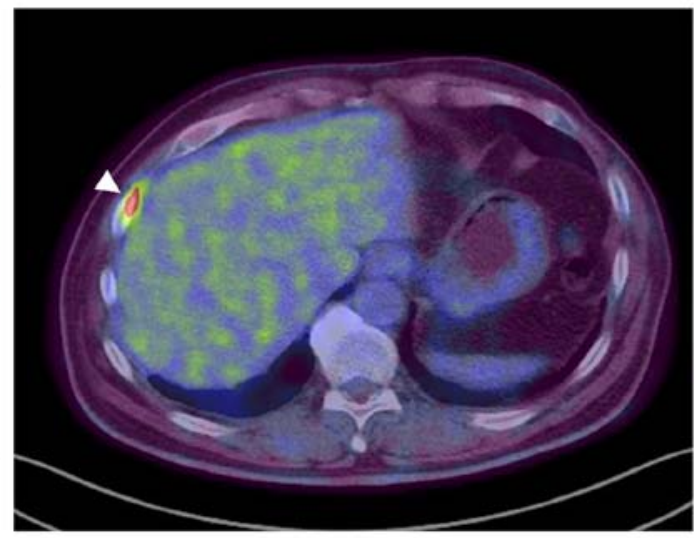

D

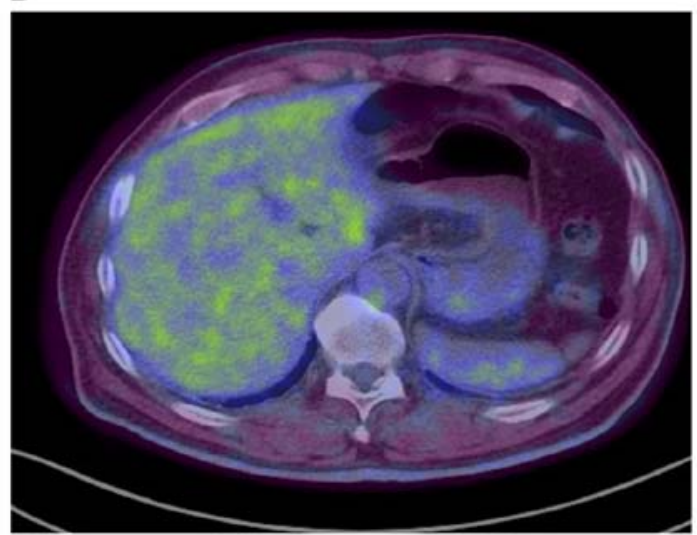

Figure 2. Response to EGFR-TKI therapy as determined by the fluorodeoxyglucose uptake (arrowheads) on positron emission tomography/CT in a case shown as 'wild-type' on cobas v2 but as 'L858R + G863D compound mutation' on direct sequencing. Prior to EGFR-TKI therapy, the pathological diagnosis was adenocarcinoma with T2aN2M0 stage IIIA after the surgery. (A) and (B) were performed prior to TKI therapy. The SUL was (A) 5.9 in the right supraclavicular lymph node and (B) 6.9 in the right pleural dissemination prior to therapy. (C and D) These SUL values declined with EGFR-TKI therapy. SUL, lean body mass corrected standardized uptake value; TKI, tyrosine kinase inhibitor.

\section{Discussion}

Previous studies have shown that PCR methods are unable to detect $10 \%$ of all EGFR mutations (4-6). In the present study, the rate of undetectable mutations was $9.4 \%(3 / 32)$ when using cobas v2, and this result was similar to that reported by previous studies (4-6). Among the undetectable mutations with cobas v2, the L858R + G863D compound mutation was detected by direct sequencing, and was identified as a single L858R mutation by Scorpion-ARMS; this was a patient with invasive adenocarcinoma, a cancer type that was solid enough to be analyzed by PCR methods. It was found that L858R was selected in cobas v2 and Scorpion-ARMS methods, while G863D was not selected in either method. It was suggested that the detection of L858R depended on the difference in the LOD between cobas v2 and Scorpion-ARMS. Moreover, the LOD of L858R in cobas v2 was 4.0-5.3 when using the cobas ${ }^{\circledR}$ EGFR Mutation Test v2 for in vitro diagnostic use, as per the description provided by Roche Diagnostics, whereas that in Scorpion-ARMS was 1.26, as shown in the therascreen ${ }^{\circledR}$ EGFR RGQ PCR Kit Handbook from Qiagen, Inc.

The reason why only Scorpion-ARMS could detect L858R in this case was likely because the LOD of L858R in Scorpion-ARMS was lower than that in cobas v2.
Mutations that were detected by direct sequencing but not by Scorpion-ARMS in the present study, including H773_V774 ins TH in exon 20, L833V, H835L and G863D in exon 21, were not selected by Scorpion-ARMS, which is why this method was unable to detect these mutations. The cases with H773_V774 ins $\mathrm{TH}$ in exon 20 and $\mathrm{L} 833 \mathrm{~V}+\mathrm{H} 835 \mathrm{~L}$ compound mutation in exon 21 did not develop recurrence. Thus, the efficacy of EGFR-TKIs against these mutations was not assessable.

Recently, next generation sequencing (NGS) has been used for the comprehensive detection of gene mutations. The types of selected genes and mutations depend on each NGS package. Moreover, NGS is not a type of whole genome or exon sequencing (12). FoundationOne CDx is one of the most widely used NGS methods, and its LOD for EGFR mutations is $2.4-5.1 \%$, as per the 'Technical Information' description provided by Foundation Medicine, Inc.

In a previous study, the frequency of compound mutations, including L858R, was found to be higher than other types of mutations, and compound mutations have properties that may be associated with poor clinical outcome compared with simple mutations (13). The present study identified two compound mutations, including L858R, and one that was detected by direct sequencing was identified as a single L858R mutation by Scorpion-ARMS (Table II). It was suggested that some 
cases with a single L858R mutation identified by selective PCR methods may harbor a compound mutation containing L858R. Furthermore, a previous study revealed that NSCLC cases with tumors harboring exon 19 deletions had a longer survival compared with those harboring L858R during EGFR-TKI therapy (4). In this previous study, the response in L858R cases may include those with a L858R compound mutation.

A previous post hoc analysis combining phase III studies suggested that second-generation EGFR-TKIs were effective in patients with uncommon mutations, particularly those involving point mutations or duplications in exons 18-21 (5). Cases with uncommon mutations in the present study, including undetectable mutations by cobas v2, such as the L858R + G863D compound mutation, showed a response to second-generation EGFR-TKI therapy. In the present study, among 32 patients with EGFR mutations, 3 patients received EGFR-TKI treatment for recurrence after surgery. Of those 3 patients, 1 patient partially responded to osimertinib; 1 patient was treated with gefitinib, but was not assessable due to the adverse events; and 1 patient responded to afatinib. The statistical significance of findings such as overall response in the present study was difficult to analyze due to the limited sample size.

The results of the present study suggested that EGFR testing using a selective method may result in patients with uncommon mutations, including those with compound mutations, missing the opportunity to receive EGFR-TKI therapy. As there are numerous variants of EGFR mutations, it is difficult to evaluate the response rate to EGFR-TKI therapy, with regards to each uncommon mutation. However, there is the possibility that cases with these mutations will respond to treatment. However, direct sequencing, including that of whole exons 18-21, is not appropriate for clinical use in all patients with NSCLC, due to the long detection time and high cost. Thus, a suitable screening test should be compatible with diverse EGFR mutations, including compound mutations, and have a lower LOD for EGFR-TKI therapy.

In conclusion, the present study demonstrated that certain mutations that were not selected by PCR methods were detectable by direct sequencing, including cases of mutations that responded to EGFR-TKI therapy. A suitable screening test should be compatible with diverse EGFR mutations, in order to avoid losing the opportunity of patients with uncommon mutations to receive EGFR-TKI therapy.

\section{Acknowledgements}

Not applicable.

\section{Funding}

The authors' department received research grants from Boehringer-Ingelheim, Chugai Pharmaceutical, Astra Zeneca, Pfizer, Taiho Pharmaceutical, MSD, Eli Lilly, Ono Pharmaceutical, Teijin and Nihon Mediphysics.

\section{Availability of data and materials}

The datasets used and/or analyzed during the current study are available from the corresponding author on reasonable request.

\section{Authors' contributions}

EN and NI conceived and designed the study; TMa, HN and TMi performed analysis of DNA data; SO, IT and KF performed the interpretation of clinical data; YM performed the pathological diagnosis; TMa, EN and TO performed the interpretation of DNA data. EN and TMa confirm the authenticity of the raw data. All authors have participated in the writing of the manuscript. All authors have read and approved the final version of the manuscript.

\section{Ethics approval and consent to participate}

The present study obtained ethics approval from the Institutional Review Board of Tokyo Medical University (approval no. 17-56), and patients provided written consent to participate.

\section{Patient consent for publication}

Not applicable.

\section{Competing interests}

The authors declare that they have no competing interests and they had no association with the promotion of PCR methods or EGFR-TKI therapy. The author's department received research grants from Boehringer-Ingelheim, Chugai Pharmaceutical, Astra Zeneca, Pfeizer, Taiho Pharmaceutical, MSD, Eli Lilly, Ono Pharmaceutical, Teijin and Nihon Mediphysics.

\section{References}

1. Yoshioka H, Shimokawa M, Seto T, Morita S, Yatabe Y, Okamoto I, Tsurutani J, Satouchi M, Hirashima T, Atagi S, et al: Final overall survival results of WJTOG3405, a randomized phase III trial comparing gefitinib versus cisplatin with docetaxel as the first-line treatment for patients with stage IIIB/IV or postoperative recurrent EGFR mutation-positive non-small-cell lung cancer. Ann Oncl 30: 1978-1984, 2019.

2. Wu YL, Cheng Y,Zhou X, Lee KH, Nakagawa K, Niho S, Tsuji F, Linke R, Rosell R, Corral J, et al: Dacomitinib versus gefitinib as first-line treatment for patients with EGFR-mutation-positive non-small-cell lung cancer (ARCHER 1050): A randomized, open-label, phase 3 trial. Lancet Oncol 18: 1454-1466, 2017.

3. Soria JC, Ohe Y, Vansteenkiste J, Reungwetwattana T, Chewaskulyong B, Lee KH, Dechaphunkul A, Imamura F, Nogami N, Kurata T, et al: Osimertinib in untreated EGFR-mutated advanced non-small cell lung cancer. N Engl J Med 378: 113-125, 2018.

4. Yang JC, Wu YL, Schuler M, Sebastian M, Popat S, Yamamoto N, Zhou C, Hu CP, O'Byrne K, Feng J, et al: Afatinib versus cisplatin-based chemotherapy for EGFR mutation-positive lung adenocarcinoma (LUX-Lung 3 and LUX-Lung 6): Analysis of overall survival data from two randomized, phase 3 trials. Lancet Oncol 16: 141-151, 2015.

5. Yang JC, Sequist LV, Geater SL, Tsai CM, Mok TS, Schuler M, Yamamoto N, Yu CJ, Ou SI, Zhou C, et al: Clinical activity of afatinib in patients with advanced non-small-cell lung cancer harbouring uncommon EGFR mutations: A combined post-hoc analysis of LUX-Lung 2, LUX-Lung 3, and LUX-Lung 6. Lancet Oncl 16: 830-838, 2015.

6. Nakajima E, Sugita M, Furukawa K, Takahashi H, Uchida O, Kawaguchi Y, Ohira T, Matsubayashi J, Ikeda N, Hirsch FR and Franklin WA: Frequency and significance of epidermal growth factor receptor mutations detected by PCR methods on patients with non-small cell lung cancer. Oncol Lett 17: 5125-5131, 2019.

7. Kimura H, Ohira T, Uchida O, Matsubayashi J, Shimizu S, Nagao T, Ikeda N and Nishio K: Analytical performance of the cobas EGFR mutation assay for Japanese non-small-cell lung cancer. Lung Cancer 83: 329-333, 2014. 
8. Nicola T, Stephen M, Antonio S, James B and Tom B: Mode of action and application of Scorpion primers to mutation detection. Nucleic Acids Res 28: 3752-3761, 2000.

9. Eisenhauer EA, Therasse P, Bogaerts J, Schwartz LH, Sargent D, Ford R, Dancey J, Arbuck S, Gwyther S, Mooney $\mathrm{M}$, et al: New response evaluation criteria in solid tumors: Revised RECIST guideline (version 1.1). Eur J Cancer 45: 228-247, 2009.

10. Richard L, Heather J, Yvette K and Martin A: From RECIST to PERCIST: Evolving considerations for PET response criteria in solid tumors. J Nucl Med 50 (Suppl 1): 122S-150S, 2009.

11. Tahari AK, Chien D, Azadi JR and Wahl RL: Optimum lean body formulation for correction of standardized uptake value in PET imaging. J Nucl Med 55: 1481-1484, 2014.
12. Umbarger MA, Kennedy CJ, Saunders P, Breton B, Chennagiri N, Emhoff J, Greger V, Hallam S, Maganzini D, Micale C, et al: Next-generation carrier screening. Genet Med 16: 132-140, 2014.

13. Kim EY, Cho EN, Park HS, Hong JY, Lim S, Youn JP, Hwang SY and Chang YS: Compound EGFR mutation is frequently detected with co-mutations of actionable genes and associated with poor clinical outcome in lung adenocarcinoma. Cancer Biol Ther 17: 237-245, 2016.

(i)(2) This work is licensed under a Creative Commons Attribution-NonCommercial-NoDerivatives 4.0 International (CC BY-NC-ND 4.0) License. 